\title{
Reliability-Aware Energy Management for Periodic Real-Time Tasks*
}

\author{
Dakai Zhu \\ Department of Computer Science \\ University of Texas at San Antonio \\ San Antonio, TX, 78249 \\ dzhu@cs . utsa . edu
}

\author{
Hakan Aydin \\ Department of Computer Science \\ George Mason University \\ Fairfax, VA 22030 \\ aydinecs.gmu .edu
}

\begin{abstract}
The prominent energy management technique, Dynamic Voltage and Frequency Scaling (DVFS), was recently shown to have direct and adverse effects on system reliability. In this work, we investigate static and dynamic reliability-aware energy management schemes for a set of periodic real-time tasks to minimize energy consumption while preserving system reliability. Focusing on EDF scheduling, we first show that the static problem is NP-hard and propose two task-level utilizationbased heuristics. Then, we develop a job-level dynamic (on-line) scheme by building on the idea of wrappertasks, to monitor and manage dynamic slack efficiently in reliability-aware settings. Our schemes incorporate recovery tasks/jobs into the schedule as needed for reliability preservation, while still using the remaining slack for energy savings. Simulation results show that all the proposed schemes can achieve significant energy savings while preserving the system reliability. Moreover, the energy savings of the static heuristics are close to those of the static optimal solution by a margin of $5 \%$.
\end{abstract}

\section{Introduction}

The phenomenal improvements in the performance of computing systems have resulted in drastic increases in power densities. For battery-operated devices with limited energy budget, energy is now considered a first-class system resource. One common strategy to save energy is to run the system components at low-performance operation points, whenever possible. For example, DVFS scales down the CPU frequency and supply voltage simultaneously to save energy [24].

For real-time systems where tasks have stringent timing constraints, scaling down the clock frequency (processing speed) may cause deadline misses and special provisions are needed. In recent past, several research studies explored the problem of minimizing energy con-

\footnotetext{
*The research of Hakan Aydin was supported by NSF CAREER Award CNS-0546244.
}

sumption while meeting all the deadlines for various realtime task models. These include a number of power management schemes which exploit the available static and/or dynamic slack in the system [3, 19, 21].

Reliability and fault tolerance have always been major factors in computer system design. Due to the effects of hardware defects, electromagnetic interferences and/or cosmic ray radiations, faults may occur at run-time, especially in systems deployed in dynamic/vulnerable environments. With the continued scaling of CMOS technologies and reduced design margins for higher performance, it is expected that, in addition to the systems that operate in electronics-hostile environments (such as those in outer space), practically all digital computing systems will be remarkably vulnerable to transient faults [8].

The backward error recovery techniques, which restore the system state to a previous safe state and repeat the computation, can be used to tolerate transient faults [20]. It is worth noting that both DVFS and backward recovery techniques are based on (and compete for) the active use of the system slack. Thus, there is an interesting trade-off between energy efficiency and the system reliability. Moreover, DVFS has been shown to have a direct and adverse effect on the transient fault rates, especially for those induced by cosmic ray radiations [29], further complicating the problem. Hence, for safety-critical realtime embedded systems (such as satellite and surveillance systems) where reliability is as important as energy efficiency, reliability-cognizant energy management becomes a necessity.

Until recently, only a few studies investigated the implications of having both fault tolerance and energy efficiency requirements $[7,18,23,25]$. As an initial study, we previously proposed a reliability-aware power management $(R A-P M)$ scheme that dynamically schedules a recovery job at task dispatch time, hence preserving the system reliability [26]. The scheme is further extended to multiple tasks with a common deadline [27]. However, preemptive scheduling, which is common for periodic real-time tasks, has not been considered.

In this work, we investigate both static and dy- 
namic RA-PM schemes for a set of periodic real-time tasks scheduled by the preemptive Earliest-DeadlineFirst (EDF) policy. Specifically, we consider the problem of exploiting the spare CPU capacity for energy savings while preserving the system reliability. We show that the optimal static RA-PM problem is NP-hard and propose two efficient heuristics for selecting a subset of tasks to use the spare capacity for the objectives of energy and reliability management. Moreover, we develop a job-level dynamic RA-PM algorithm that monitors and manages the dynamic slack which may be generated at run-time, again for these dual objectives. The latter algorithm is built on the wrapper-task mechanism: the key idea is to conserve the dynamic slack allocated to scaled tasks for recovery across preemption points, which is essential for preserving reliability. To the best of our knowledge, this is the first research effort that provides a comprehensive energy management framework for periodic real-time tasks while preserving the system reliability.

The remainder of this paper is organized as follows. The models and problem formulation are presented in Section 2. Section 3 focuses on the task-level, utilizationbased static RA-PM schemes. The wrapper-task concept is introduced and the job-level dynamic RA-PM scheme is presented in Section 4. Simulation results are presented and discussed in Section 5. We conclude in Section 6.

\section{System Model and Problem Description}

\subsection{Application Model}

We consider a set of independent periodic real-time tasks $\Gamma=\left\{T_{1}, \ldots, T_{n}\right\}$. The task $T_{i}$ is characterized by a pair $\left(p_{i}, c_{i}\right)$, where $p_{i}$ represents its period and $c_{i}$ denotes its worst case execution time (WCET). The $j^{\text {th }}$ job of $T_{i}$, which is referred to as $J_{i j}$, arrives at time $(j-1) \cdot p_{i}$ and has a deadline of $j \cdot p_{i}$.

In DVFS settings, it is assumed that the WCET $c_{i}$ of task $T_{i}$ is given under the maximum processing speed $f_{\max }$. For simplicity, we assume that the execution time of a task scales linearly with the processing speed ${ }^{1}$. That is, at speed $f$, the execution time of task $T_{i}$ is assumed to be $c_{i} \cdot \frac{f_{\max }}{f}$.

The system utilization is defined as $U=\sum_{i=1}^{n} u_{i}$, where $u_{i}=\frac{c_{i}}{p_{i}}$ is task $T_{i}$ 's utilization. The tasks are to be executed on a uni-processor system according to the preemptive EDF policy. Considering the well-known feasibility condition for EDF [17], we assume that $U \leq 1$.

\footnotetext{
${ }^{1} \mathrm{~A}$ number of studies have indicated that the execution time of tasks does not scale linearly with reduced processing speed due to accesses to memory [22] and/or I/O devices [4]. However, exploring the full implications of this observation is beyond the scope of this paper and is left as our future work.
}

\subsection{Power Model}

The relation between the supply voltage and operating frequency is known to be almost linear [5]. DVFS reduces supply voltages for lower frequencies [24] and we will use the term frequency change to stand for both supply voltage and frequency adjustments. Considering the ever-increasing static leakage power due to scaled feature size and increased levels of integration [15] as well as the power-saving states provided in modern powerefficient components (e.g., CPU [2] and memory [16]), in this work, we adopt the simple system-level power model proposed in [29], where the power consumption $P$ of a computing system is given by:

$P=P_{s}+\hbar\left(P_{i n d}+P_{d}\right)=P_{s}+\hbar\left(P_{i n d}+C_{e f} f^{m}\right)$

Despite its simplicity, this power model captures the essential components for system-wide energy management. Here, $P_{s}$ is the static power, which includes the power to maintain basic circuits and keep the clock running. It can be removed only by powering off the whole system. $P_{\text {ind }}$ is the frequency-independent active power, which is a constant and corresponds to the power that is independent of CPU processing speed. It can be efficiently removed by putting systems into sleep state(s) $[2,16] . P_{d}$ is the frequency-dependent active power, which includes processor's dynamic power and any power that depends on system processing speeds $[5,16]$.

When there is computation in progress, the system is active and $\hbar=1$. Otherwise, when the system is turned off or in power-saving sleep modes, $\hbar=0$. The effective switching capacitance $C_{e f}$ and the dynamic power exponent $m$ (in general, $2 \leq m \leq 3$ [5]) are systemdependent constants. $f$ is the normalized processing frequency with $f_{\max }=1$.

Intuitively, when executing a given job, lower frequencies result in less frequency-dependent active energy consumption. But with reduced speeds, the job runs longer and thus consumes more static and frequencyindependent active energy. Therefore, a minimal energyefficient frequency $f_{e e}$, below which DVFS starts to consume more total energy, does exist [12, 15, 21]. Considering that energy is the integral of power over time, from the above equation, one can find that $^{2}$ [29]:

$$
f_{e e}=\sqrt[m]{\frac{P_{i n d}}{C_{e f} \cdot(m-1)}}
$$

Consequently, for energy efficiency, we assume that $f_{e e} \leq f \leq f_{\max }$. We develop our framework by assuming continuous frequency. The implications of having discrete speed levels are discussed in Section 5.3.

${ }^{2}$ Considering the prohibitive overhead of turning on/off a system (e.g., tens of seconds), we assume that the system will not be turned off during the interval considered and $P_{s}$ is always consumed. 


\section{$2.3 \quad$ Fault Model}

At run-time, faults may occur due to various reasons, such as hardware failures, electromagnetic interferences as well as the effects of cosmic ray radiations. The transient faults occur much more frequently than permanent faults [14], especially with the continued scaling of CMOS technologies and reduced design margins [8]. Consequently, in this paper, we focus on transient faults, and use backward recovery techniques for fault tolerance. It is assumed that the faults are detected using sanity (or consistency) checks at the completion of a job's execution, and if needed, the recovery task is dispatched, in the form of re-execution [20].

In our previous work [29], we have studied the negative effects of DVFS on transient faults induced by cosmic ray radiations. Assuming that transient faults follow Poisson distribution [25], the average transient fault rate for systems running at frequency $f$ (and corresponding supply voltage) can be expressed as [29]:

$$
\lambda(f)=\lambda_{0} \cdot g(f)
$$

where $\lambda_{0}$ is the average fault rate corresponding to $f_{\max }$. That is, $g\left(f_{\max }\right)=1$. With reduced processing speeds and supply voltages, fault rate generally increases [29]. Therefore, we have $g(f)>1$ for $f<f_{\max }$.

\subsection{Problem Description}

Our primary objective in this paper is to develop power management schemes for periodic real-time tasks executing on a uni-processor system and preserve system reliability at the same time. We define the reliability of a real-time job as the probability of its being correctly executed before its deadline. One of the key findings reported in [29] is that the reliability of a job whose execution is scaled through DVFS decreases drastically due to the increased fault rates and extended execution time.

The reliability of a real-time system depends on the correct execution of all jobs within their deadlines. Without loss of generality, we assume that the system reliability is satisfactory when no power management scheme is applied, even under the worst-case scenario (i.e., when jobs take their WCETs). To preserve system reliability, for simplicity, we focus on maintaining the reliability of individual jobs in this work. Specifically, for a periodic real-time task set with utilization $U$, we consider the problem of how to use the spare CPU utilization $(1-U)$, as well as the dynamic slack generated at run-time, for maximizing energy savings while keeping the reliability of any job of task $T_{i}$ no less than $R_{i}^{0}$ $(i=1, \ldots, n)$, where $R_{i}^{0}=e^{-\lambda_{0} c_{i}}$ (from Poisson fault arrival pattern and the average fault rate $\lambda_{0}$ [26]) is the original reliability of $T_{i}$ 's jobs, when there is no power management and the jobs use their WCETs.

\subsection{Reliability-Aware Power Manage- ment (RA-PM)}

Conventionally, DVFS-based ordinary power management schemes exploit all the available (static and/or dynamic) slack for energy management and are, consequently, reliability-ignorant (in the sense that no attention is paid to the potential effects of DVFS on task reliabilities). Instead of using all the available slack for DVFS to save energy, one can reserve a portion of the slack to schedule a recovery job $R J$ for any job $J$ whose execution is scaled down, to recuperate the reliability loss due to the energy management [26]. The recovery job $R J$ will be dispatched (at the maximum frequency $f_{\max }$ ) only if a transient fault is detected when $J$ completes. The recovery can be in the form of re-execution and $R J$ has the same WCET as that of $J$ [20].

With the help of $R J$, the overall reliability $R$ of job $J$ will be the summation of the probability of $J$ being executed correctly and the probability of having transient fault(s) during $J$ 's execution while the recovery job $R J$ being executed correctly. We have shown that, if the amount of available slack is more than the WCET of a job, by scheduling a recovery job (e.g., re-execution), one can guarantee to preserve the reliability of a realtime job while still obtaining energy savings using the remaining slack, regardless of different fault rate increases and scaled processing speeds [26]. In increasing level of sophistication and implementation complexity, we first introduce the task-level static schemes and then job-level dynamic schemes in the next two sections.

\section{Task-Level Static Schemes}

To start with, we consider static RA-PM schemes that make their decisions at the task-level. In this approach, for simplicity, all the jobs of a task have the same treatment. That is, if a given task is selected for energy management, all its jobs will run at the same scaled frequency; otherwise, they will run at $f_{\max }$. From the above discussion, to recuperate reliability loss due to scaled execution, each scaled job ${ }^{3}$ will need a corresponding recovery job within its deadline, should a fault occur.

To provide the required recovery jobs, we can construct periodic recovery tasks $(R T)$ by exploiting the spare CPU capacity (or, static slack). The recovery task will have the same timing parameters (i.e., WCET and period) as those of the task to be scaled. Hence, we can schedule a recovery job for each primary job and preserve its reliability. Note that a recovery job will be activated only when the corresponding primary job incurs a fault.

As a concrete example, suppose that we have a periodic task set of three tasks $\Gamma=\left\{T_{1}(1,7), T_{2}(2,14)\right.$,

\footnotetext{
${ }^{3}$ We use the expression scaled job to refer to any job whose execution is slowed down through DVFS, for energy management purposes.
} 


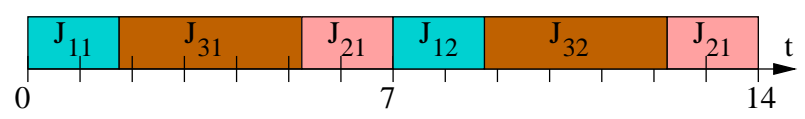

a. the optimal ordinary SPM

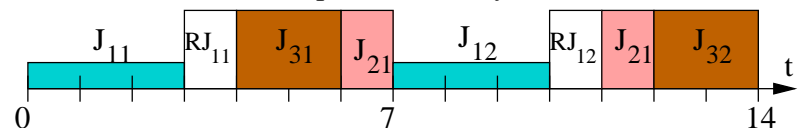

b. task-level RA-PM when managing task $T_{1}$

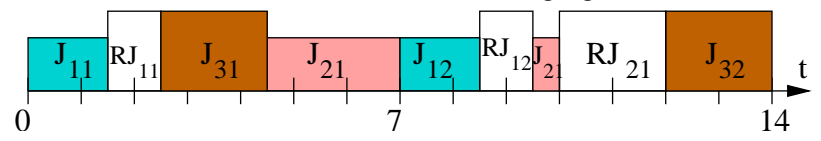

c. task-level RA-PM when managing tasks $T_{1}$ and $T_{2}$

\section{Figure 1. Static schemes for a task set with three tasks $\left\{T_{1}(1,7), T_{2}(2,14), T_{3}(2,7)\right\}$.}

$\left.T_{3}(2,7)\right\}$ with system utilization as $U=\frac{4}{7}$. Without considering system reliability, the optimal ordinary static power management (SPM) under EDF will scale down all tasks at the speed $f=U \cdot f_{\max }=\frac{4}{7}$ as shown in Figure 1a [3, 19]. In the figure, the $\mathrm{X}$-axis represents time and the height of task boxes represents processing speed. Due to the periodicity, only the schedule within the least common multiple (LCM) of tasks' periods is shown. However, by uniformly scaling down the execution in this way, the reliability figures of all the tasks (and that of the system) would be significantly reduced [29].

When applying static RA-PM, we first compute the spare capacity as $1-U=\frac{3}{7}$. After constructing the recovery task $R T_{1}(1,7)$, which has the same WCET and period as the task $T_{1}$ with the utilization $r u_{1}=\frac{1}{7}$, the overall system utilization will be $U^{\prime}=U+r u_{1}=\frac{5}{7}$. If we allocate the remaining spare capacity (i.e., $1-U^{\prime}=$ $\frac{2}{7}$ ) to task $T_{1}$, all jobs of $T_{1}$ can be executed at the speed of $\frac{1}{3}$. With the recovery task $R T_{1}$ and the scaled execution of $T_{1}$, the effective system utilization is exactly 1 and the modified task set is schedulable under EDF as shown in Figure 1b. From the figure, we can see that every scaled job of task $T_{1}$ has a corresponding recovery job within its deadline. Therefore, all $T_{1}$ 's jobs could preserve their reliability level $R_{1}^{0}$. Since the jobs of tasks $T_{2}$ and $T_{3}$ run at $f_{\max }$, their reliability figures are preserved at the levels of $R_{2}^{0}$ and $R_{3}^{0}$, respectively.

Therefore, by incorporating a recovery task for the task to be managed, the task-level utilization-based static RA-PM scheme could preserve system reliability while obtaining energy savings. In [27], we reported that it is not optimal (in terms of energy savings) for the RA-PM scheme to utilize all the slack for a single task in case of aperiodic tasks. Similarly, we can use the spare capacity for multiple periodic tasks for better energy savings. For instance, Figure 1c shows the case where both $T_{1}$ and $T_{2}$ are scaled to speed $\frac{2}{3}$ after constructing the recovery tasks $R T_{1}$ and $R T_{2}$. For illustration purposes, we assume that the system power is given by a cubic function. Simple algebra shows that, managing only task $T_{1}$ could save $\frac{8}{9} E$, where $E$ is the energy consumed by all jobs of task $T_{1}$ within LCM under no power management. In comparison, the energy savings would be $\frac{11}{9} E$ if both $T_{1}$ and $T_{2}$ are managed, which is a significant improvement.

\subsection{Intractability of Task-Level RA-PM}

The inherent complexity of the optimal static RA-PM problem warrants an analysis. Suppose that the system utilization of the task set is $U$ and the spare capacity is $s c=1-U$. If a subset $\Phi$ of tasks are selected for management with total utilization $X=\sum_{T_{i} \in \Phi} u_{i}<s c$, after accomodating all recovery tasks, the remaining spare capacity (i.e., $s c-X$ ) could be used to scale down the selected tasks for energy management. Considering the convex relation between power and processing speed (see Equation 1), the solution that minimizes the energy consumption will uniformly scale down all jobs of the selected tasks, where the scaled processing speed will be $f=\frac{X}{X+(s c-X)}=\frac{X}{s c}$. Therefore, without considering the energy consumed by recovery jobs, the amount of total fault-free energy consumption within $L C M$ would be:

$$
\begin{aligned}
E_{L C M}= & L C M \cdot P_{s}+L C M(U-X)\left(P_{i n d}+c_{e f} \cdot f_{\text {max }}^{m}\right) \\
& +L C M \cdot s c\left(P_{\text {ind }}+c_{e f} \cdot\left(\frac{X}{s c}\right)^{m}\right)
\end{aligned}
$$

where the first part is the energy consumption due to static power, the second part captures the energy consumption of unselected tasks, and finally, the third part represents the energy consumption of the selected tasks. Simple algebra shows that, when $X_{o p t}=s c$. $\left(\frac{P_{i n d}+C_{e f}}{m \cdot C_{e f}}\right)^{\frac{1}{m-1}}, E_{L C M}$ will be minimized.

If $X_{\text {opt }} \geq U$, all tasks should be scaled down appropriately to minimize energy consumption. Otherwise, the problem becomes essentially a task selection problem, where the summation of the selected tasks' utilization should be exactly equal to $X_{o p t}$, if possible. In other words, such a choice would definitely be the optimal solution.

Theorem 1 For a set of periodic tasks, the problem of the task-level utilization-based static RA-PM is NP-hard.

Proof We consider a special case of the problem with $m=2, C_{e f}=1$ and $P_{i n d}=0$; that is, $X_{o p t}=\frac{s c}{2}$. We show that even this special instance is intractable, by transforming the PARTITION problem, which is known to be NP-hard [10], to that special case.

In PARTITION, the objective is to find whether it is possible to partition a set of $n$ integers $a_{1}, \ldots, a_{n}$ (where $\sum_{i=1}^{n} a_{i}=S$ ) into two disjoint subsets, such that the sum of numbers in each subset is exactly $\frac{S}{2}$.

Given an instance of the PARTITION problem, we construct the corresponding static RA-PM instance as follows: we have $n$ periodic tasks, where $c_{i}=a_{i}$ and $p_{i}=2 \cdot S$. Note that, in this case, $U=\sum \frac{c_{i}}{p_{i}}=\frac{1}{2}$, 
$s c=1-U=\frac{1}{2}$. Observe that, the energy savings will be maximized if it is possible to find a subset of tasks whose total utilization is exactly $X_{o p t}=\frac{s c}{2}=\frac{1}{4}$. Since $p_{i}=2 S \forall i$, this is possible if and only if one can find a subset of tasks $\Phi$ such that $\sum_{i \in \Phi} c_{i}=\frac{S}{2}$. But this can happen only if the original PARTITION problem admits a YES answer. Therefore, if RA-PM problem had a polynomial-time solution, one could also solve the PARTITION problem in polynomial-time, by constructing the corresponding RA-PM problem, and checking if the maximum energy savings that can be obtained correspond to the amount we could gain through managing exactly $X_{o p t}=\frac{s c}{2}=25 \%$ of the periodic workload.

\subsection{Heuristics for Task-Level RA-PM}

Considering the intractability of the problem, we propose two simple heuristics for selecting tasks for energy management: Largest-utilization-first (LUF) and Smallest-utilization-first (SUF). Suppose that the tasks in a given periodic task set are indexed in the nondecreasing order of their utilizations (i.e., $u_{i} \leq u_{j}$ for $1 \leq i<j \leq n$ ). SUF will select the first $k$ tasks, where $k$ is the largest integer that satisfies $\sum_{i=1}^{k} u_{i} \leq X_{\text {opt }}$. Similarly, LUF will select the last $k$ tasks, where $k$ is the smallest integer that satisfies $\sum_{i=k}^{n} u_{i} \leq X_{\text {opt }}$.

Here, SUF tries to manage as many tasks as possible, since any managed jobs could achieve better reliability. However, at some point, when the remaining spare capacity is not enough to accomodate a recovery task for the task with the next smallest utilization, SUF may waste significant portion of the spare capacity. LUF tries to select larger utilization tasks first, where the amount of wasted spare capacity is at most the smallest utilization among all tasks. The potential drawback of LUF is that, sometimes, relatively few tasks might be managed for energy savings. These heuristics are evaluated in Section 5.

\section{Job-Level Dynamic RA-PM}

In our backward recovery framework, the recovery jobs are executed only if their corresponding scaled jobs fail. Otherwise, the CPU time reserved for recovery jobs is freed and becomes dynamic slack at run-time. Moreover, it is well-known that real-time tasks typically take a small fraction of their WCETs [9]. Therefore, significant amount of dynamic slack can be expected at run time, which should be exploited to further save energy and/or enhance system reliability.

Unlike the greedy RA-PM scheme which allocates all available dynamic slack for the next ready task when the tasks share a common deadline [26], in periodic execution settings, the run-time dynamic slack will be generated at different priorities and may not be always reclaimable by the next ready job [3]. Moreover, possible preemptions that a job experiences after it has reclaimed some slack further complicates the problem. This is because, once a job's execution is scaled through DVFS, additional slack must be reserved for potential recovery operations to preserve system reliability. Hence, maintaining the reclaimed slack until the job completes successfully is essential in reliability-aware settings.

The slack management problem has been studied extensively (e.g., CASH-queue [6] and $\alpha$-queue [3] approaches) for different purposes. By borrowing and also extending some fundamental ideas from these studies, we provide a new framework which guarantees the conservation of the reclaimed slack, thereby maintaining the reliability figures.

Specifically, in this work, we propose the wrappertask mechanism to track/manage dynamic slack. For any dynamic slack generated at run-time, a new wrapper-task will be created with the following two timing parameters: a size that equals the amount of dynamic slack generated and a deadline that is equal to that of the job whose early completion gave rise to this slack. A wrapper-task is destroyed when all the slack it represents is reclaimed or wasted. Otherwise, it will compete for CPU along with normal real-time jobs. When a wrapper-task has the highest priority (i.e., the earliest deadline) and is "scheduled", it will "fetch" the highest priority job in the ready queue (if any) and wrap the job's execution during the interval when the wrapper-task is "executed". If there is ready job, the CPU will become idle, the wrapper-task is said to "execute no-ops" and the corresponding dynamic slack is consumed/wasted during this time interval.

\subsection{An Example with Wrapper-Tasks}

Before formally presenting the algorithm, we first illustrate the idea of wrapper-tasks through a detailed example. We consider a task-set with four periodic real-time tasks $\Gamma=\left\{T_{1}(1,6), T_{2}(6,10), T_{3}(2,15)\right.$, $\left.T_{4}(3,30)\right\}$. For jobs within $L C M(=30)$, suppose that $J_{21}, J_{22}, J_{23}$ and $J_{41}$ take $2,3,4$ and $2 \frac{1}{3}$ time units, respectively, and all other jobs take their WCETs.

Recall that EDF scheduling is used. For jobs with the same deadline, the one with the smaller task index is assumed to have higher priority. When $J_{21}$ completes early at time 3,4 units of dynamic slack will be generated and the system state is shown in Figure 2a. Here, a wrappertask (shown as dotted rectangle) is created to represent the slack, which is labeled by two numbers: a size (e.g., 4) and a deadline (e.g., 10). Similar to ready jobs that are kept in the ready queue (Ready-Q) (where the deadlines are indicated by the numbers at the bottom of the job boxes), wrapper-tasks are kept in a WT-Queue in increasing order of their deadlines.

It is known that, the slack that a job $J_{x}$ can reclaim (i.e. the reclaimable slack) should have a deadline no later than $J_{x}$ 's deadline [3]. From our previous discussion, to recuperate reliability loss due to energy management, 


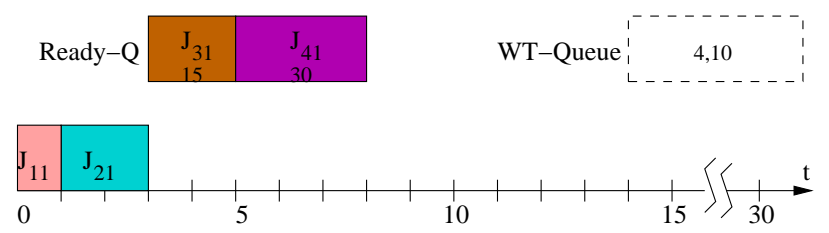

a. $J_{21}$ completes early at time 3
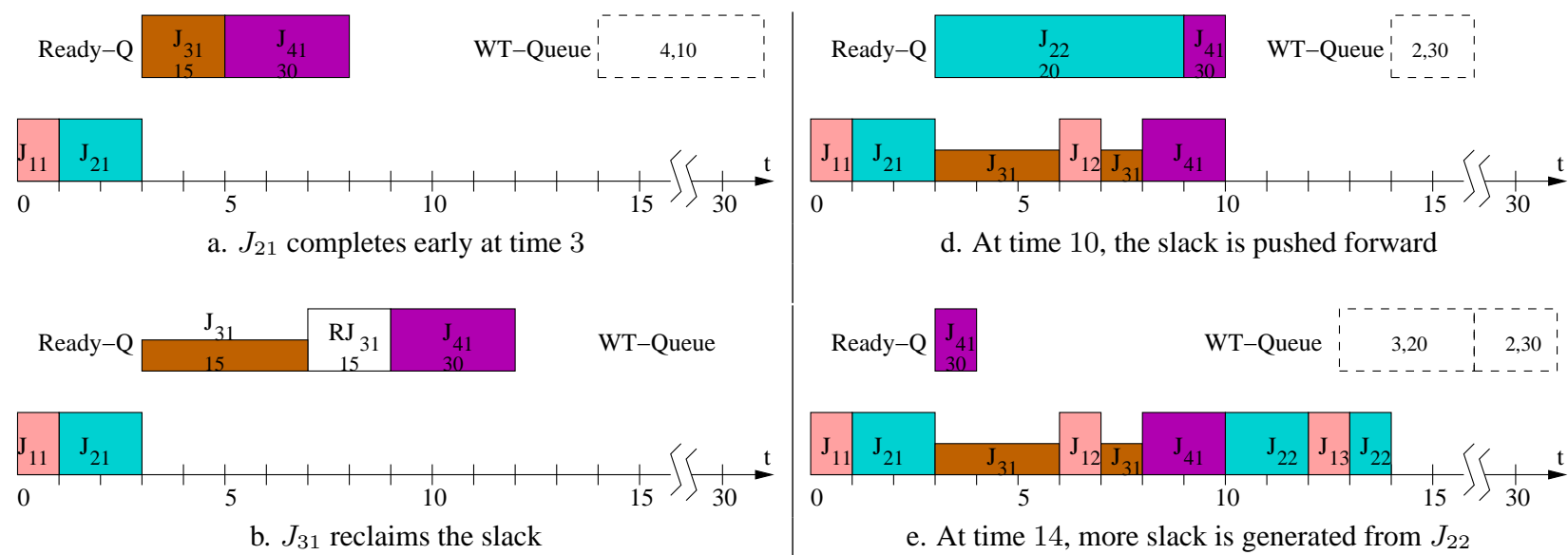

d. At time 10, the slack is pushed forward

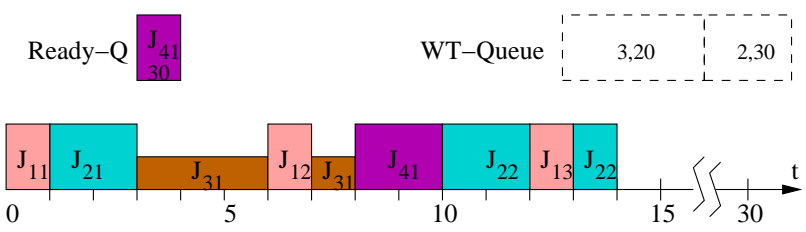

e. At time 14 , more slack is generated from $J_{22}$
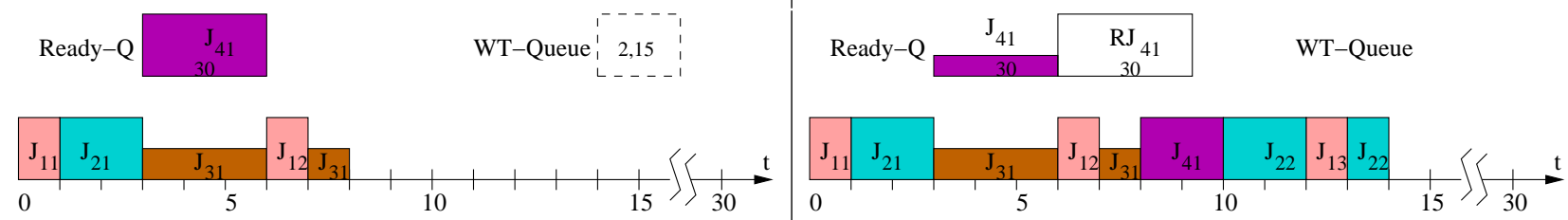

f. Partial job $J_{41}$ is scaled and needs a full recovery job $R J_{41}$

c. Scaled $J_{31}$ finishes correctly, $R J_{31}$ freed as slack
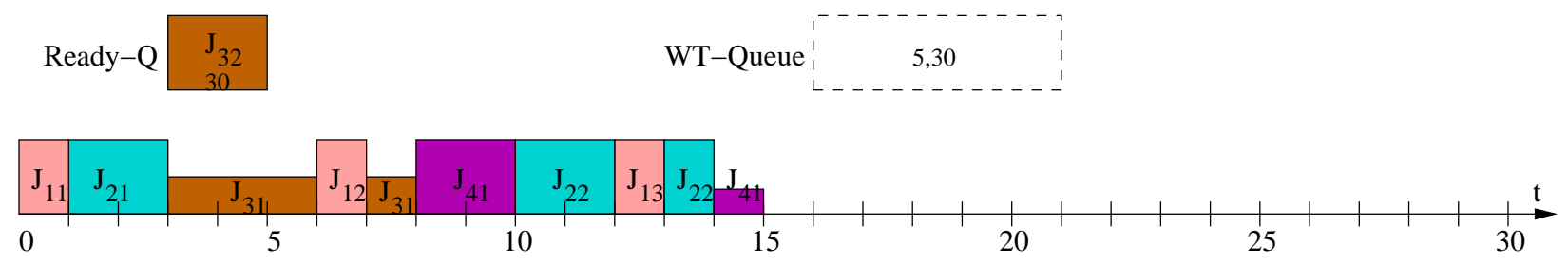

g. Scaled $J_{41}$ finishes early, both its remaining time and $R J_{41}$ are released as slack at time $15, J_{32}$ arrives

$$
\begin{array}{c|c|c|c|c|c}
\hline \text { Ready-Q } & \mathrm{J}_{15} & \mathrm{~J}_{32} & \mathrm{RJ}_{32} \\
30 & 30 & 30
\end{array} \quad \text { WT-Queue } \begin{array}{cc} 
& 2,30 \\
\hline
\end{array}
$$

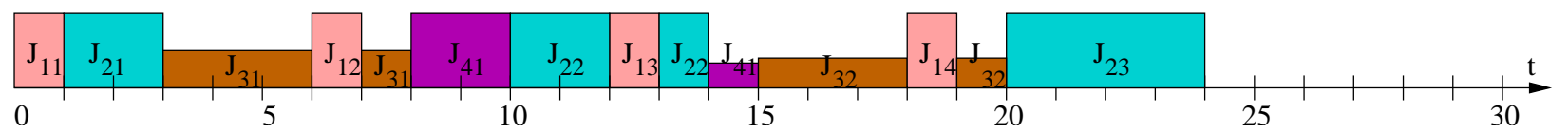

h. Scaled $J_{32}$ is preempted (but its reclaimed slack is conserved) and more slack is generated from $J_{22}$ at time 24

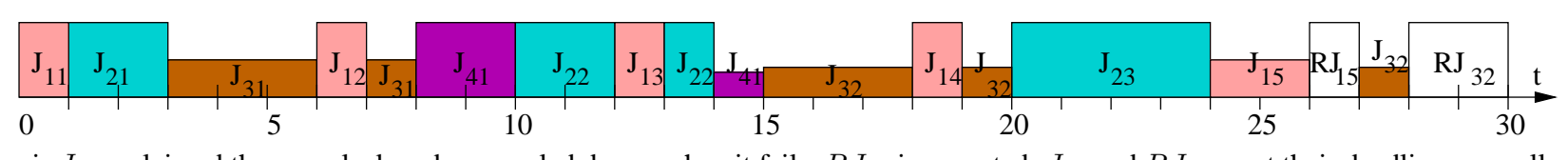

i. $J_{15}$ reclaimed the new slack and was scaled down; when it fails, $R J_{15}$ is executed; $J_{32}$ and $R J_{32}$ meet their deadlines as well

Figure 2. Using wrapper-tasks to manage dynamic slack

a recovery job needs to be scheduled within $J_{x}$ 's deadline. Hence, a non-scaled job will reclaim the slack only if the amount of reclaimable slack is larger than the job size. Thus, at time $3, J_{31}$ reclaims the available slack and scales down its execution as shown in Figure 2b. Here, a recovery job $R J_{31}$ is created. The scaled execution of $J_{31}$ uses the time slots of the reclaimed slack and is scaled at speed $\frac{2}{4}=\frac{1}{2}$, while $R J_{31}$ will take $J_{31}$ 's original time slots. Both $J_{31}$ and $R J_{31}$ could finish their execution within $J_{31}$ 's deadline in the worst case.

Suppose that the scaled $J_{31}$ finishes its execution correctly at time 8 , after being preempted by $J_{12}$ at time 6 . The recovery job $R J_{31}$ will be removed from $\operatorname{Ready}-Q$ and all its time slots will become slack as shown in Figure 2c. But this slack is not sufficient for reclamation by $J_{41}$. However, since the corresponding wrapper-task has higher priority, it is scheduled and wraps the execution of $J_{41}$. When the wrapper-task finishes at time 10 , a 
new wrapper-task with the same size is created, but with the deadline of $J_{41}$. It can also be viewed as $J_{41}$ borrowing the slack for its execution and returning it with the extended deadline (i.e., the slack is pushed forward). The schedule and queues at time 10 , after $J_{22}$ arrives, are shown in Figure 2d.

When $J_{22}$ completes early at time 14 (after being preempted by $J_{13}$ at time 12), 3 units of slack is generated with the deadline of 20, as shown in Figure 2e. Now, we have two pieces of slack (represented by two wrappertasks, respectively) with different deadlines.

Note that, as faults are assumed to be detected at the end of a job's execution, a full recovery job is needed to recuperate the reliability loss due to even partially scaled execution ${ }^{4}$. Thus, when the partially-executed $J_{41}$ reclaims all the available slack (since both wrapper-tasks have deadlines no later than $J_{41}$ 's deadline), a full recovery job $R J_{41}$ is created and inserted into Ready-Q. $J_{41}$ uses the remaining slack to scale down its execution appropriately as shown in Figure $2 \mathrm{f}$.

When the scaled $J_{41}$ finishes early at time 15 , both its unused CPU time and $R J_{41}$ are freed as slack. After the arrival of $J_{32}$ at time 15 , the schedule and queues are shown in Figure $2 \mathrm{~g}$. Here, $J_{32}$ will reclaim the slack and be scaled to speed $\frac{2}{5}$ after reserving the slack for the recovery job $R J_{32}$. After the scaled $J_{32}$ is preempted by $J_{14}$ and $J_{23}$, at time 18 and 20, respectively, and $J_{23}$ completes early at time 24 , Figure $2 \mathrm{~h}$ shows the newly generated slack and the state of Ready- $Q$, which contains $J_{15}$ (with arrival time 24). Note that, the recovery job $R J_{32}$ (i.e., the slack time) is conserved even after $J_{32}$ is preempted by higher priority jobs.

$J_{15}$ reclaims the new slack. Suppose that both of the scaled jobs $J_{15}$ and $J_{32}$ fail, then, $R J_{15}$ and $R J_{32}$ will be executed as illustrated in Figure 2i. It can be seen that all jobs (including recovery jobs) finish their executions on time and no deadline is missed.

\subsection{Job-level RA-DPM Algorithm}

As the example illustrated, in addition to $\operatorname{Ready}-Q$ that is used to hold the ready jobs, a wrapper-task queue (i.e., WT-Queue) is needed to track/manage available dynamic slack. The rules for managing dynamic slack with wrapper-tasks are as follows:

- Rule 1 (slack generation): When new slack is generated due to early completion of jobs or removal of recovery jobs, a new wrapper-task is created. However, it may be merged with an existing element in WT-Queue if they have the same deadline. That is, all wrapper-tasks in WT-Queue represent slack with different deadlines. Wrapper-tasks in WT-Queue are kept in the increasing order of their deadlines.

\footnotetext{
${ }^{4}$ Although checkpointing could be used for partial recovery [25], we have shown that checkpoints with a single recovery section cannot guarantee to preserve task reliability [26].
}

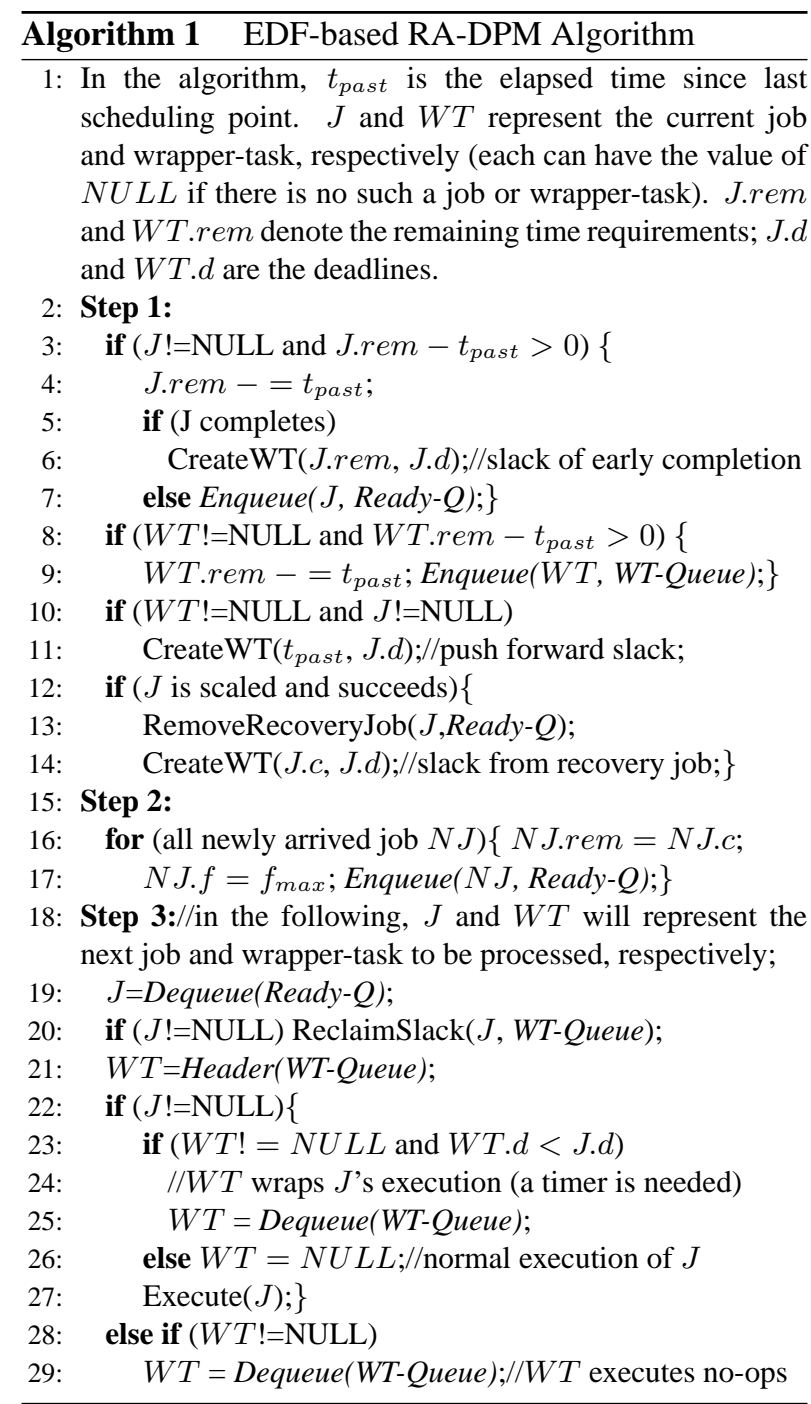

- Rule 2 (slack reclamation): The slack is reclaimed when: (a) a non-scaled job has the highest priority in Ready- $Q$ and its reclaimable slack is larger than the job size; or (b) the highest priority job in Ready$Q$ has been scaled (i.e., its recovery job has been reserved) but its speed is higher than $f_{e e}$ and there is reclaimable slack. After the slack is reclaimed, the corresponding wrapper-tasks are removed from WT-Queue and destroyed.

- Rule 3 (slack forwarding/wasting): the wrappertasks of non-reclaimed slack compete for CPU along with ready jobs. When a wrapper-task has higher priority (i.e., earlier deadline) and wraps the execution of a job, the corresponding slack is pushed forward; otherwise, if a wrapper-task executes no-ops, the corresponding slack is wasted. Note that, when wrapped execution is interrupted by higher priority jobs, only part of slack (which is consumed by the wrapped execution) will be pushed forward, while the remaining part has the original deadline. 
The outline of the EDF-based RA-DPM algorithm is shown in Algorithm 1. Note that, RA-DPM may be invoked by three types of events: job arrival, job completion and wrapper-task completion (a timer can be used to signal a wrapper-task's completion to operating system). As common routines, we use Enqueиe $(J, Q)$ to add a job/wrapper-task to the corresponding queues and, Dequeue $(Q)$ to fetch the highest priority (i.e., the header) job/wrapper-task and remove it from the queue. Moreover, Header $(Q)$ is used to retrieve the header job/wrapper-task without removing it from the queue.

At each scheduling point, as the first step (from line 3 to line 14), the remaining execution time information of the currently running job and wrapper-task (if any) are updated. If they did not complete, they are put back to Ready-Q and WT-Queue (lines 7 and 9), respectively. When a wrapper-task (WT) is used and wraps the execution of $J$ (line 11), as discussed before, the corresponding amount of slack (i.e., $t_{\text {past }}$ ) is pushed forward by creating a new wrapper-task with the deadline of the currently wrapped job. Otherwise, the slack is consumed (wasted).

If the current job completes early (line 6 ) or its recovery job is removed due to the primary job's successful scaled execution (lines 13 and 14), new slack is generated and corresponding wrapper-tasks are created.

Secondly, if new jobs arrive at the current scheduling point, they are added to Ready- $Q$ according to their EDF priorities (line 17). The remaining timing requirements will be set as their WCETs at the speed $f_{\max }$. The last step is to choose the next highest priority ready job $J$ (if any) for execution (lines 19 to 29 ). $J$ first tries to reclaim the available slack (line 20; details are shown in Algorithm 2). Then, depending on the priority of the remaining wrapper-tasks, the execution of $J$ may be wrapped by a wrapper-task (line 25) or executed normally (line 26). When a wrapper-task has the highest priority but no job is ready, the wrapper-task executes no-ops (line 29).

Algorithm 2 further shows the details of slack reclamation. As mentioned previously, recovery jobs are executed at $f_{\max }$ and are not scaled (line 1). For a job $J$, by traversing WT-Queue, we can find out the amount of reclaimable slack (lines 3 and 5 ). If $J$ is not a scaled job (i.e., its recovery job is not reserved yet) and the amount of reclaimable slack is no larger than the size of $J$ (i.e., $J . c)$, the available slack is not enough for reclamation (line 7). Otherwise, after properly reserving the slack for recovery (line 8), $J$ 's new speed is calculated, which is bounded by $f_{e e}$ (line 9; as discussed in Section 2). The actual amount of slack used by $J$ includes those for energy management (line 10) as well as the slack for recovery job (where the recovery job is created and added to Ready- $Q$ in line 12). For the reclaimed slack, the corresponding wrapper-task(s) will be removed from WTQueue and destroyed (lines 15 to 20), which ensures that this slack is conserved for the scaled job, even if higher-

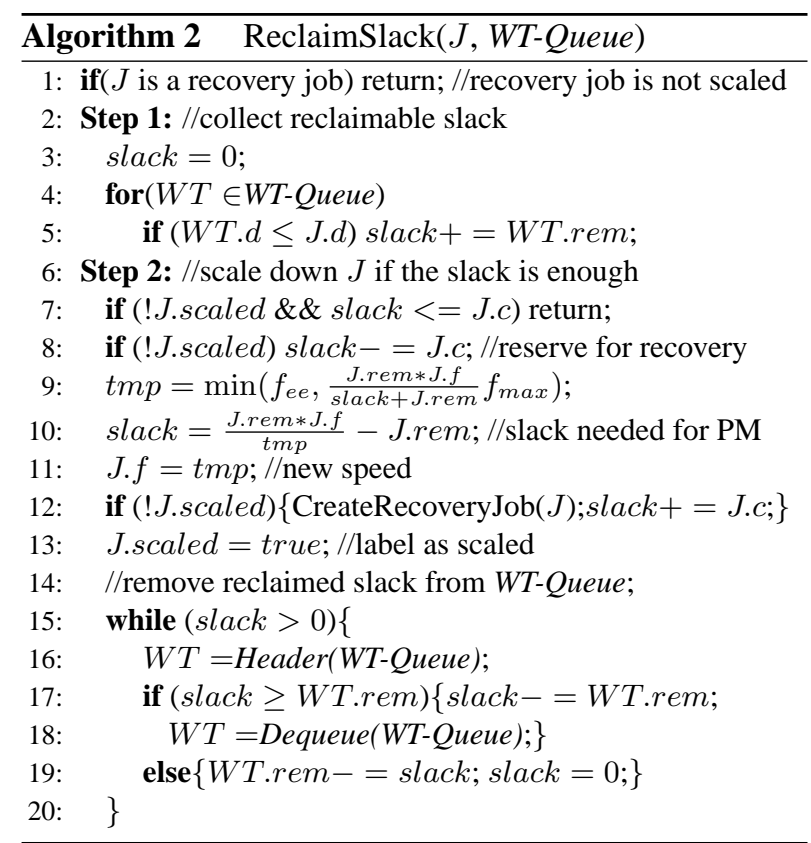

priority jobs preempt the scaled job's execution later.

\subsection{Analysis of RA-DPM}

Note that, when all jobs in a task set present their WCETs at run time, there will be no dynamic slack and no wrapper-task will be created. In this case, RA-DPM will perform the same as EDF and generate the same worst case schedule, which is feasible by assumption. However, as some jobs complete early, RA-DPM will undertake slack reclamation and/or wrapped execution, and one needs to show that the feasibility is preserved even after these changes in CPU time allocation of jobs.

Theorem 2 For a periodic real-time task set whose utilization does not exceed $100 \%$, the feasibility of the task set is preserved under RA-DPM.

The full formal proof is omitted due to space limitations, but can be found in [28]. To sketch the proof, first, we observe that, the elements of WT-Queue represent the slack of tasks that complete early. These slack elements, while being reclaimed, may be entirely or partially re-transformed to actual workload. Our strategy consists in proving that, at any time $t$ during execution, the remaining workload could be feasibly scheduled by $E D F$, even if all the slack elements in WT-Queue were to be re-introduced to the system, with their corresponding deadlines and remaining worst-case execution times (sizes). This, in turn, allows us to show the feasibility of the actual schedule, since the above-mentioned property implies the feasibility even with an over-estimation of the actual workload, for any time $t$.

Regarding the time complexity, note that the deadlines of wrapper-tasks correspond to real-time jobs' deadlines. 
At any time $t$, there are at most $n$ different deadlines corresponding to jobs with release times on or before $t$ and deadlines on or after $t$. That is, the number of wrappertasks in WT-Queue is at most $n$. Therefore, slack reclamation can be performed (by traversing WT-Queue) in time $O(n)$. Hence, the complexity of RA-DPM is $O(n)$ at each scheduling point.

\section{Simulation Results and Discussion}

To evaluate the performance of our proposed schemes, we developed a discrete event simulator using $\mathrm{C}++$. In the simulations, we consider six different schemes. First, the scheme of no power management (NPM), which executes all tasks/jobs at $f_{\max }$ and puts system to sleep states when idle, is used as the baseline for comparison. The ordinary static power management (SPM) scales all tasks uniformly at speed $f=U \cdot f_{\max }$ (where $U$ is the system utilization). For the task-level static RA-PM, after obtaining the optimal utilization $\left(X_{o p t}\right)$ that should be managed, two heuristics are considered: smaller utilization task first (RA-SPM-SUF) and larger utilization task first (RA-SPM-LUF). For dynamic schemes, we implemented our job-level dynamic RA-PM (RA-DPM) and the cycle conserving $E D F(C C-E D F)$ [19], a well-known but reliability-ignorant DVFS algorithm.

We focus on active power and assume $P_{\text {ind }}=0.1$, $C_{e f}=1$ and $m=3$. The energy efficient frequency is $f_{e e}=0.37$ (see Section 2). Transient faults are assumed to follow the Poisson distribution with an average fault rate of $\lambda_{0}=10^{-6}$ at $f_{\max }$ (and corresponding supply voltage), which corresponds to 100,000 FITs (failure in time, in terms of errors per billion hours of use) per megabit and is a reasonable fault rate as reported [11, 30]. To take the effects of DVFS on fault rates into consideration, we adopt the exponential fault model developed in [29] and assume that the exponent $d=2$. That is, the average fault rate is assumed to be 100 times higher at the lowest speed $f_{e e}$ (and corresponding supply voltage). The effects of different values of $d$ were evaluated in our previous work [26, 27, 29].

We consider synthetic real-time task sets where each task set contains 20 periodic tasks. The periods of tasks $(p)$ are uniformly distributed within the range of $[10,20]$ (for short-period tasks) or [20, 200] (for longperiod tasks). The WCETs of tasks are uniformly distributed in the range of 1 and their periods. Finally, the WCETs of tasks are scaled by a constant such that the system utilization of tasks reaches a desired value [19]. The variability in the actual workload is controlled by the $\frac{W C E T}{B C E T}$ ratio (that is, the worst-case to best-case execution time ratio), where the actual execution time of tasks follows a normal distribution with mean and standard deviation being $\frac{W C E T+B C E T}{2}$ and $\frac{W C E T-B C E T}{6}$, respectively [3].
We simulate the task set's execution for $10^{7}$ and $10^{8}$ time units, for short- and long-period task sets, respectively. That is, approximately 20 million jobs are executed during each run. Moreover, for each result point in the graphs, 100 task sets are generated and the presented results correspond to the average.

\subsection{Performance of Task-Level Schemes}

For different system utilization (i.e., spare capacity), we first evaluate the performance of the task-level static schemes. It is assumed that all jobs take their WCETs. Figure 3a first shows the probability of failure (i.e., 1 -reliability) for NPM and static schemes for task sets with short periods (i.e., $p \in[10,20]$ ). Here, the probability of failure shown is the ratio of the number of failed jobs over the total number of jobs executed.

From the figure, we can see that, as system utilization increases, for NPM, the probability of failure increases slightly. The reason for this is that, with increased total utilization, the computation requirement for each task increases and tasks run longer, which increases the probability of being subject to transient fault(s). The probability of failure for SPM increases dramatically due to increased fault rates and extended execution time. Note that, the minimum energy efficient frequency is $f_{e e}=$ 0.37. For very low system utilization (i.e., $U<0.37$ ), SPM executes all tasks with $f_{e e}$. The probability of failure for SPM increases slightly with increased utilization for the same reason as NPM. However, when system utilization is higher than 0.37 , the processing speed of SPM increases with increased utilization, which has lower failure rates and results in decreased probability of failure.

For reliability-aware SPM schemes (i.e., RA-SPMSUF and RA-SPM-LUF), by incorporating a recovery task for each task to be scaled, the probability of failure is lower than that of NPM and system reliability is preserved, which confirms the theoretical result obtained in Section 3. Note that, with 20 tasks in a task set, the utilization for each task is a small number and is typically close to each other. Therefore, RA-SPM-SUF and RA-SPM-LUF perform roughly the same.

The probability of failure for long-period task sets is shown in Figure 3b, where all schemes have similar behavior to that of short-period task sets. However, for the same system utilization, long-period task sets will have longer execution time (almost 10 times longer), which leads to a probability of failure which is roughly 10 times greater.

Figure $3 c$ further shows the normalized energy consumption for short-period tasks with NPM as a baseline. Here, reliability-aware SPM schemes consume roughly $20 \%$ more energy than that of ordinary SPM because there is less spare capacity available for energy management. Moreover, the figure also shows the energy consumption for $O P T-B O U N D$, which is calculated as the 


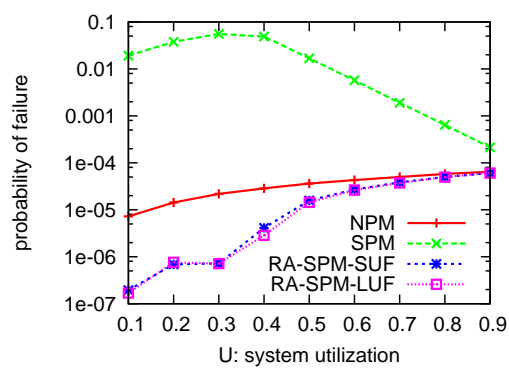

a. reliability for $p \in[10,20]$

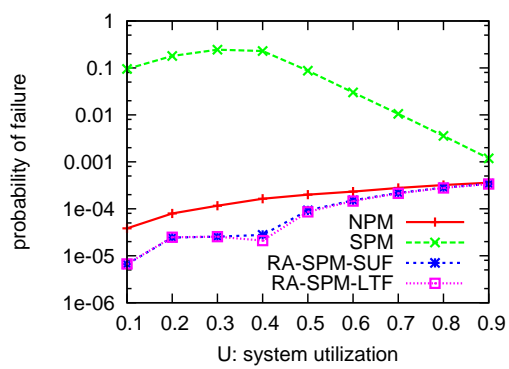

b. reliability for $p \in[20,200]$

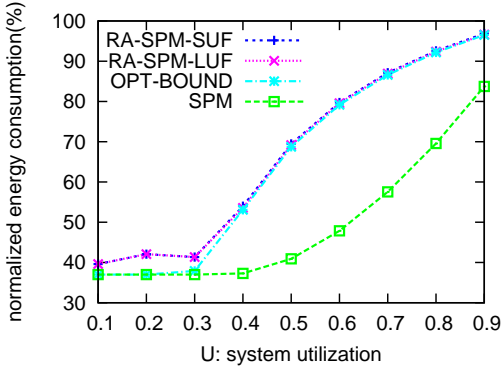

c. energy for $p \in[10,20]$

Figure 3. Reliability and energy for static schemes.

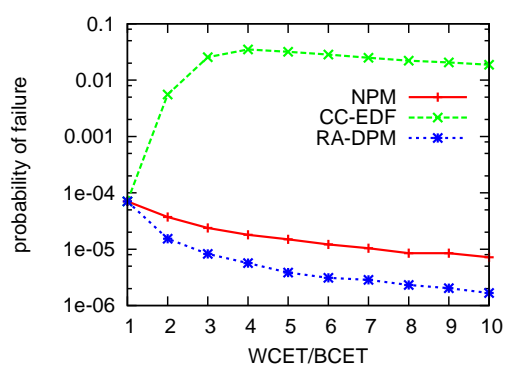

a. reliability for $p \in[10,20]$

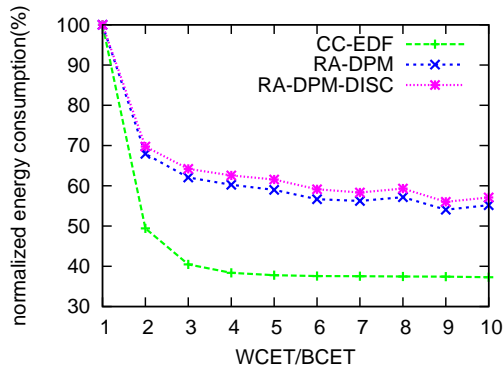

b. energy for $p \in[10,20]$

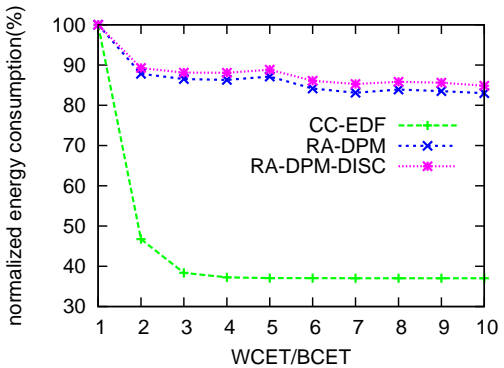

c. energy for $p \in[20,200]$

Figure 4. Reliability and energy for dynamic schemes.

fault-free energy consumption with the assumption that the managed tasks have the accumulated utilization $e x$ actly equal to $X_{\text {opt }}$. Clearly, OPT-BOUND provides a performance bound for the optimal static solution. Thus, from the figure, we can see that the normalized energy consumption for two heuristics will be within $5 \%$ of that for the optimal solution. For long-period tasks, the normalized results are similar, and are not included due to space limitations.

\subsection{Performance of Job-Level Schemes}

With system utilization being fixed at $U=1.0$, we vary $\frac{W C E T}{B C E T}$ ratio and evaluate the performance of the dynamic schemes. Figure 4a first shows the probability of failure for short-period tasks. Here, we can see that, as $\frac{W C E T}{B C E T}$ ratio increases, more dynamic slack is available. The probability of failure for CC-EDF first decreases radically due to scaled execution, then decreases slightly because of shorter execution time. Again, by reserving slack for recovery jobs, RA-DPM preserves system reliability. The results for long-period tasks are similar.

Figure $4 \mathrm{~b}$ shows the normalized energy consumption for short-period tasks. Initially, as the ratio of $\frac{W C E T}{B C E T}$ increases, more dynamic slack is available and normalized energy consumption decreases. Due to limitation of $f_{e e}$, when $\frac{W C E T}{B C E T}>5$, the normalized energy consumption for both schemes stays roughly the same and RA-DPM consumes about $15 \%$ more energy than CCEDF. RA-DPM performs much worse than CC-EDF for long period tasks (in terms of energy) as shown in Fig- ure 4c. One possible explanation could be that, when slack is pushed forward excessively by the long-period tasks, this may prevent other jobs from reclaiming, and it may be wasted.

\subsection{Effects of Discrete Speeds}

So far, we have assumed that the clock frequency can be scaled continuously. However, current DVFS-enabled processors (e.g., Intel XScale [1]) only have a few speed levels. Nevertheless, our schemes can be easily adapted to discrete speed settings. After obtaining the desired speed (e.g., Algorithm 2 line 9), we can either use two adjacent frequency levels to emulate the task's execution at that speed [13], or use the next higher discrete speed to ensure the algorithm's feasibility. Assuming Intel XScale model [1] with 5 speed levels $\{0.15,0.4,0.6,0.8,1.0\}$ and using the next higher speed, we re-ran the simulations. The results for normalized energy consumption are represented as RA-DPM-DISC and shown in Figure 4bc. Here, the cases for discrete speeds consume at most $2 \%$ more energy than that of continuous speed. The reason is that, with the next higher discrete speed, the unused slack is not wasted but actually saved for future usage.

\section{Conclusions}

DVFS was recently shown to have negative impact on settings where transient faults become more prominent with continued scaling of CMOS technologies and reduced design margins. In this work, we proposed for the 
first time a reliability-aware energy management (RAPM) framework for periodic tasks. Focusing on EDF scheduling, we first studied task-level utilization-based static RA-PM schemes that exploit the system spare capacity. We showed the intractability of the problem and proposed two efficient heuristics. Moreover, we proposed the wrapper-task mechanism for efficiently managing dynamic slack and presented a job-level dynamic RA-PM scheme. The scheme is able to conserve the slack reclaimed by a scaled job, which is an essential requirement for reliability preservation, across preemption points.

The proposed schemes are evaluated through simulations with synthetic real-time workloads. The results show that, compared to those of ordinary energy management schemes, the new schemes could achieve significant amount of energy savings while preserving system reliability. However, ordinary energy management schemes that are reliability-ignorant, often lead to drastically decreased system reliability.

\section{References}

[1] Intel xscale technology and processors. http://developer.intel.com/design/intelxscale/.

[2] Intel corp. mobile pentium iii processor-m datasheet. Order Number: 298340-002, Oct 2001.

[3] H. Aydin, R. Melhem, D. Mossé, and P. Mejia-Alvarez. Dynamic and aggressive scheduling techniques for poweraware real-time systems. In Proc. of IEEE Real-Time Systems Symposium, 2001.

[4] E. Bini, G. Buttazzo, and G. Lipari. Speed modulation in energy-aware real-time systems. In Proc. of the $17^{\text {th }}$ Euromicro Conference on Real-Time Systems, 2005.

[5] T. D. Burd and R. W. Brodersen. Energy efficient cmos microprocessor design. In Proc. of The HICSS Conference, Jan. 1995

[6] M. Caccamo, G. Buttazzo, and L. Sha. Capacity sharing for overrun control. In Proc. of Real-Time Systems Symposium, 2000.

[7] E. M. Elnozahy, R. Melhem, and D. Mossé. Energyefficient duplex and tmr real-time systems. In Proc. of The $23^{\text {rd }}$ IEEE Real-Time Systems Symposium, Dec. 2002.

[8] D. Ernst, S. Das, S. Lee, D. Blaauw, T. Austin, T. Mudge, N. S. Kim, and K. Flautner. Razor: circuit-level correction of timing errors for low-power operation. IEEE Micro, 24(6):10-20, 2004.

[9] R. Ernst and W. Ye. Embedded program timing analysis based on path clustering and architecture classification. In Proc. of The Int'l Conference on Computer-Aided Design, pages 598-604, 1997.

[10] M. R. Garey and D. S. Johnson. Computers and Intractability: A Guide to the Theory of NP-Completeness. Mathematical Sciences Series. Freeman, 1979.

[11] P. Hazucha and C. Svensson. Impact of cmos technology scaling on the atmospheric neutron soft error rate. IEEE Trans. on Nuclear Science, 47(6):2586-2594, 2000.

[12] S. Irani, S. Shukla, and R. Gupta. Algorithms for power savings. In Proc. of The $14^{\text {th }}$ Symposium on Discrete Algorithms, 2003.

[13] T. Ishihara and H. Yauura. Voltage scheduling problem for dynamically variable voltage processors. In Proc. of The
Int'l Symposium on Low Power Electronics and Design, 1998.

[14] R. Iyer, D. J. Rossetti, and M. Hsueh. Measurement and modeling of computer reliability as affected by system activity. ACM Trans. on Computer Systems, 4(3):214-237, Aug. 1986.

[15] R. Jejurikar, C. Pereira, and R. Gupta. Leakage aware dynamic voltage scaling for real-time embedded systems. In Proc. of the $41^{\text {st }}$ Design automation conference, 2004

[16] A. R. Lebeck, X. Fan, H. Zeng, and C. S. Ellis. Power aware page allocation. In Int'l Conference on Architectural Support for Programming Languages and Operating Systems, 2000.

[17] C. L. Liu and J. W. Layland. Scheduling algorithms for multiprogramming in a hard real-time environment. $J$. ACM, 20(1):46-61, 1973.

[18] R. Melhem, D. Mossé, and E. M. Elnozahy. The interplay of power management and fault recovery in real-time systems. IEEE Trans. on Computers, 53(2):217-231, 2004.

[19] P. Pillai and K. G. Shin. Real-time dynamic voltage scaling for low-power embedded operating systems. In Proc. of $18^{\text {th }}$ ACM Symposium on Operating Systems Principles, Oct. 2001.

[20] D. K. Pradhan. Fault Tolerance Computing: Theory and Techniques. Prentice Hall, 1986.

[21] S. Saewong and R. Rajkumar. Practical voltage scaling for fixed-priority rt-systems. In Proc. of the $9^{\text {th }}$ IEEE RealTime and Embedded Technology and Applications Symposium, 2003.

[22] K. Seth, A. Anantaraman, F. Mueller, and E. Rotenberg. Fast: Frequency-aware static timing analysis. In Proc. of the $24^{\text {th }}$ IEEE Real-Time System Symposium, 2003.

[23] O. S. Unsal, I. Koren, and C. M. Krishna. Towards energyaware software-based fault tolerance in real-time systems. In Proc. of The Int'l Symposium on Low Power Electronics Design, 2002.

[24] M. Weiser, B. Welch, A. Demers, and S. Shenker. Scheduling for reduced cpu energy. In Proc. of The First USENIX Symposium on Operating Systems Design and Implementation, Nov. 1994.

[25] Y. Zhang and K. Chakrabarty. Energy-aware adaptive checkpointing in embedded real-time systems. In Proc. of IEEE/ACM Design, Automation and Test in Europe Conference(DATE), 2003.

[26] D. Zhu. Reliability-aware dynamic energy management in dependable embedded real-time systems. In Proc. of the IEEE Real-Time and Embedded Technology and Applications Symposium, 2006.

[27] D. Zhu and H. Aydin. Energy management for real-time embedded systems with reliability requirements. In Proc. of the International Conference on Computer Aidded Design (ICCAD), Nov. 2006.

[28] D. Zhu and H. Aydin. Reliability-aware energy management for periodic real-time tasks. Technical report, Dept. of Computer Science, Univ. of Texas at San Antonio, 2006. available at http://www.cs.utsa.edu/ dzhu/papers/zhu-tr-2007a.pdf.

[29] D. Zhu, R. Melhem, and D. Mossé. The effects of energy management on reliability in real-time embedded systems. In Proc. of the Int'l Conference on Computer Aidded Design, 2004.

[30] J. F. Ziegler. Trends in electronic reliability: Effects of terrestrial cosmic rays. available at http://www.srim.org/SER/SERTrends.htm, 2004. 